COMMENT. The incidence of sero-positive acetylcholine receptor (AChR) binding antibodies is lower in juvenile-onset myasthenia gravis compared to adult-onset case-studies; $63 \%$ (Afifi and Bell 1993) v 85\% (Richman and Agius 2003), respectively. In a series of 30 juvenile MG cases studied in Turkey (Anlar et al, 1996) AchR antibodies were positive in $34 \%$, and response to treatment was not significantly different in seropositive $\mathrm{cf}$ seronegative cases. None of the antibody-positive cases was in remission. Myasthenic crises occurred in $33 \%$. In a current report from Mexico (Guillermo GR et al. Acta Neurologica Scandinavica March 2004;109:217-221) the response to thymectomy was evaluated in 71 patients with MG of all ages (14 [20\%] seronegative, and 57 [80\%) seropositive). The response was similar in the two groups, with no differences in prognosis between the seronegative and seropositive patients.

In the Afifi and Bell, 1993 University of Iowa series of 27 juvenile MG cases, ocular myasthenics responded to pyridostigmine alone, while generalized myasthenics required corticosteroids and/or thymectomy. Those with normal thymus had a higher rate of remission than patients with thymic hyperplasia. Thymectomized patients had a $35 \%$ remission rate. In a series of 35 juvenile myasthenics (Millichap and Dodge 1960), 43\% had respiratory difficulties, $40 \%$ of these requiring tracheostomy. Twenty-one underwent thymectomy and of these $18(86 \%)$ showed complete or partial remission. Complete remission without the need for drug therapy was obtained in $29 \%$ of thymectomized compared to $14 \%$ of patients treated only with cholinergic drugs. Rodriquez et al, in 1983, found a cumulative 15 -year remission rate of $55 \%$ following thymectomy, compared to a spontaneous remission rate of $30 \%$ in myasthenic children. The remission rate was higher following thymectomy performed within 12 months of the onset of symptoms. The reported high incidence of myasthenic crises and respiratory difficulties, and the satisfactory results of early thymectomy emphasize the importance of prompt introduction of immune-directed management when spontaneous remission is delayed.

\title{
MYOPATHY IN CRITICALLY ILL CHILDREN
}

The incidence of muscle weakness was determined in 830 children ( 3 months to 17 years of age) admitted for $>24$ hours to the intensive care unit over a 1 -year period at the Hospital for Sick Children, Toronto, Canada. Generalized weakness developed in 14 (1.7\%), and 4 failed repeated attempts to extubate. The age distribution was bimodal, with 3 under age 3 years and 11 age 10 or older. Eleven had multiple organ dysfunction and 9 had sepsis. Most received corticosteroids, neuromuscular blocking agents, or aminoglycoside antibiotics. Eight of the 14 were organ or bone marrow transplant recipients. EMG findings in 5 were abnormal and myopathic in 4, with short-duration, low-amplitude motor unit potentials and small polyphasic potentials. Nerve conduction studies done in 7 patients showed decreased compound muscle action potentials in 4 , normal findings in 2 , a compressive neuropathy in 1 , and a demyelinating polyneuropathy in 1 . Serum creatine kinase was elevated to 2 to 100 times the upper limit of normal in 3. A histological diagnosis of acute quadriplegic myopathy was made in all 3 patients with muscle biopsy. Three died; and in survivors, weakness persisted for 3 to 12 months after discharge. (Banwell BL, Mildner RJ, Hassall AC et al. Muscle weakness in critically ill children. Neurology December (2 of 2) 2003;61:17791782). (Respond: Dr BL Banwell, Department of Pediatrics (Neurology), Hospital for Sick Children, 555 University Ave, Toronto, Ontario, Canada M5G 1X8). 
COMMENT. The incidence of neuromuscular weakness in critically ill children in an ICU is infrequent and develops mainly in older children, over 10 years of age, and especially in transplant recipients.

\section{CEREBRAL PALSY}

\section{CORD SERUM INFLAMMATORY CYTOKINES AS BIOMARKERS OF CEREBRAL PALSY}

The relationship between 78 inflammatory protein mediators in cord serum and cerebral palsy (CP) in 41 term and preterm children was determined at the University of Oulu. Finland. Infants were born in the year 1992-1993 at 4 university hospitals, and CP was diagnosed by child neurologists by age 5, according to standard criteria: a persistent abnormality of muscle tone, movement, and posture with functional impairment due to a nonprogressive lesion of the immature brain. Children with congenital cerebral malformation, prenatal viral or protozoal infection, post-neonatal brain damage, or chromosomal abnormality were excluded. Of 41 children with CP in the study group, 22 had spastic diplegia, 12 spastic hemiplegia, 3 spastic quadriplegia, and 4 dystonic quadriplegia. One paired control matched for gestational age and without $\mathrm{CP}$ was selected for each case.

In children with $\mathrm{CP}$, the levels of 8 cytokines, 1 growth factor, and 3 chemokines were higher $(\mathrm{p}<0.05)$ than in controls, findings consistent with an infection or fetal inflammatory response. Infants developing $\mathrm{CP}$ had an altered serum profile of specific protein mediators. Prematures had high IL-6 and IL-8 levels, and 9 additional cytokines showed significant changes. Several of these regulatory proteins found in newborns with $\mathrm{CP}$ are distinct from those with acute neonatal disease not resulting in $\mathrm{CP}$. Inflammatory mediators and growth factors in cord blood of CP infants are indicative of a fetal response to a pre- or perinatal infection that manifests after birth as permanent brain damage. (Kaukola T, Satyaraj E, Patel DD et al. Ann Neurol February 2004;55:186-194). (Respond: Mikko Haliman MD, PhD, Department of Pediatrics and Biocenter of Oulu, University of Oulu, PO Box 5000, FIN-90014 Oulu, Finland).

COMMENT. In references cited by the authors, chorioamnionitis is considered a risk factor for cerebral palsy (Wu YW, Colford JM. JAMA 2000;284:1417-1424); and an increase in proinflammatory cytokines in amniotic fluid or cord blood has been associated with the development of CP (Nelson KB et al. Ann Neurol 1998;44:665-675). The Finnish investigators propose that susceptible infants have an abnormal pattern of cytokines and growth factors that predispose to $\mathrm{CP}$ during perinatal infection and lead to neuronal and neuroglial brain damage. An editorial (Dammann, Leviton A. Ann Neurol Feb 2004;55:158161) cautions that the study group is small, and no appreciable differences are demonstrated between cases and controls in the distribution of the "classic" proinflammatory cytokines (eg. IL-1B, IL-6, tumor necrosis factor-a). More studies of developmental regulation of biomarkers are required to adjust for gestational age-specific changes. 